\title{
Assessment of sdLDL-C by Three Different Formula and Its Correlation with Clinical Variables Among Diabetes Individuals with and without Nephropathy
}

\author{
Desy Thayyil Menambath ${ }^{1}$, Durga Rao Yalla ${ }^{1}$, Ashok Prabhu Khandige ${ }^{2}$, \\ Sudha Kuthethur ${ }^{1}$, Nandini Mangalore ${ }^{1, *}$ \\ ${ }^{1}$ Department of Biochemistry, Centre for Basic Sciences, Kasturba Medical College, Mangalore, Manipal Academy of Higher Education, \\ Manipal, Karnataka, India 575004 \\ ${ }^{2}$ Clinical Biochemistry Section, Kasturba Medical College Hospital, Ambedkar Circle, Mangalore, India 575001 \\ *Corresponding author. E-mail: nandini.m@manipal.edu
}

Received date: Jun 15, 2020; Revised date: Nov 4, 2020; Accepted date: Nov 13, 2020

\section{Abstract}

B ACKGROUND: Diabetes mellitus is a common disease worldwide which affects renal function. Cardiovascular morbidity and mortality in diabetes patients can be accelerated by dyslipidemia. Small dense lowdensity lipoprotein-cholesterol (sdLDL-C) is atherogenic and its predominance has been known as a cardiovascular risk factor. The study aimed to assess the validity of calculated sdLDL-C using three different formulae and its association with other clinical variables in diabetic patients with and without nephropathy, and also to determine the best suited formula to measure sdLDL-C.

METHODS: The study subjects were divided into two groups based on the amount of albumin excreted in the urine. Group I or the control group consisted of diabetic subjects without microalbuminuria, while group II consisted of diabetic subjects with microalbuminuria. Blood glucose, glycated haemoglobin ( $\mathrm{HbAlc}$ ), creatinine, total cholesterol (TC), triglyceride (TG), high-density lipoprotein-cholesterol (HDL-C), LDL-C and apoB were estimated. Three formulae used for the validation of calculated sdLDL-C were TG/ $\mathrm{HDL}, \mathrm{sdLDL}(\mathrm{mg} / \mathrm{dL})=0.580($ nonHDL $)+0.407$ (directLDL-C) -0.719 (calculated-LDL-C) - 12.05, and LDL-C/ LDL apoB.

RESULTS: There was no significant difference in sdLDL-C levels of diabetic subjects with and without nephropathy. The sdLDL-C had strong correlation with TC, TG, LDL-C, very-low-density lipoprotein (VLDL), non-HDL and apoB in both study groups. ROC curve showed that LDL-C/LDL apoB derived sdLDL-C had better sensitivity (85\%) and specificity (69\%) compared to other two measures.

CONCLUSION: Though the calculated sdLDL-C do not predict the occurrence of nephropathy in diabetes subjects, it may still be used in conjunction with the traditional markers since it is cost effective. The LDL-C/LDL apoB formula is the best predictor of sdLDL-C among the three equations.

KEYWORDS: HDL-C, LDL-C, Microalbuminuria, sdLDL-C, T2DM

Indones Biomed J. 2020; 13(2): 140-6

\section{Introduction}

Type 2 Diabetes mellitus (T2DM) is one of the systemic disorders affecting the kidneys. Microalbuminuria serves as predictive parameter of morbidity and mortality in cardiovascular disease (CVD), as well as in renal complications.(1) The presence of microalbumin as an independent predictor of higher morbidity and mortality has been reported.(2) It is a known predictor for progressive renal function loss, cardiovascular morbidity and mortality in patients with diabetic nephropathy. As damage to the kidneys worsens, the blood pressure rises with the increase of the plasma triglyceride (TG), apolipoproteinB (apoB) and the higher concentrations of small dense lowdensity lipoprotein-cholesterol (sdLDL) are also seen. The sdLDL particles are believed to be atherogenic and their predominance has been accepted as an emerging 
cardiovascular risk factor.(3) A study suggested that subjects with elevated sdLDL have higher TG and lower high-density lipoprotein-cholesterol (HDL-C), a finding, similar to subjects with insulin resistance.(4) Increased prevalence of sdLDL in Asian Indians has been reported. (6) Prospective and epidemiologic studies have found that microalbuminuria can be a predictive parameter of CVD mortality and coronary heart disease (CHD) events in patients with diabetes and/or hypertension.(6) The sdLDL-C, a subfraction of LDL-C, is a better marker for prediction of CVD compared to the total LDL-C.(7)

Measurement of sdLDL requires sophisticated equipment and lengthy analytical time preventing its clinical use.(8) The novel homogenous enzymatic assay was developed to measure sdLDL (9) though easier to use, yet it is highly expensive. The present study aimed to determine the utility of calculated sdLDL-C among T2DM subjects with and without nephropathy, and to determine the best calculated formula for the measure of sdLDL-C in clinical practice.

\section{Methods}

\section{Subject Recruitment}

This is a hospital based study conducted on subjects who came for routine health check-up. Their case sheets were used for collecting patients necessary details. The study included diabetic subjects aged between 50-65 years. Subjects with frank proteinuria, acute infections, haematuria, established coronary artery diseases, thyroid dysfunction and end stage renal disease were excluded from the study.

The participants were divided into two groups based on the amount of albumin excreted in urine. Thirty-two T2DM subjects with microalbuminuria $(20-200 \mathrm{mg} / \mathrm{L}$ of albumin in a spot urine sample) and 32 T2DM subjects without microalbuminuria $(<20 \mathrm{mg} / \mathrm{L}$ of albumin in a spot urine sample) constituted the case group and control group respectively.

The Ethical approval was obtained from Institutional Ethics committee, Kasturba Medical College, Mangalore (IEC KMC MLR 12-17/251) prior to the study.

\section{Measurement of Biochemical Parameters}

Five $\mathrm{mL}$ of fasting venous blood was collected. Whole blood was used for glycated haemoglobin $(\mathrm{HbAlc})$. Serum was used for estimation of glucose, creatinine lipid profile and apoB. Morning spot urine sample was used for the quantitation of albumin. HbA1c was estimated by High
Performance liquid chromatography method (10) using BioRad D10 system (BioRad Laboratories Inc., Hercules, CA, USA). Glucose, lipid profile and creatinine estimations were carried out in Cobas 6000 auto analyzer using the kits supplied by Roche Diagnostics (Mannheim, Germany) as per the manufacturer's instructions.(11-16) ApoB (apoB1) was measured by nephelometric method (17) using kits from Agappe Diagnostics. While, microalbumin was determined by the immune-turbidimetric method.(18)

Estimation of sdLDL-C was done by using previously developed equation obtained from commonly available measurements of non-HDL-C, and LDL-C.(8) The formula was: sdLDL $(\mathrm{mg} / \mathrm{dL})=0.580($ non-HDL $)+0.407$ (directLDL-C) -0.719 (calculated-LDL-C) -12.05 . This was compared with two other equations that provided an estimate of sdLDL, which were TG/HDL: ratio $>3$ predict the presence of sdLDL, a surrogate marker for sdLDL particles (19); and LDL-C/LDL apoB: ratio $<1.2$ indicate the preponderance of sdLDL (20). LDL apoB $=$ Apo B 0.09TC + 0.09 HDL-C $-0.08 \mathrm{TG}$.

\section{Statistical Analysis}

Data was analyzed using SPSS version 20 (IBM Coorporation, Armonk, NY, USA). Student's independent T-test was used to compare the difference between the two groups. Pearson's correlation analysis was done to find out the association of sdLDL-C with the various parameters in both the groups. A $p<0.05$ was considered statistically significant. The receiver operating characteristic (ROC) curve was plotted to establish cut-off values for sdLDL-C, LDL-C/LDL apoB and TG/HDL in both the groups. The sensitivity and specificity together with the area under curve (AUC) was reported.

\section{Results}

Table 1 showed the demographic data and other clinical parameters of the subjects. No significant difference was observed between age and gender. Significant increase in the levels of microalbumin was seen in subjects with nephropathy with no significant changes in the fasting blood sugar (FBS), HbAlc and the creatinine levels in both groups. There was no significant change in the lipid profile as well as apoB in diabetic subjects with and without nephropathy (Table 2).

Measurement of sdLDL-C, by the use of three different formula did not show any statistical significance in the two groups. Correlations of clinical parameters with the three 
Table 1. Demographic data and other clinical parameters of subjects.

\begin{tabular}{lccc}
\hline \multicolumn{1}{c}{ Variable } & $\begin{array}{c}\text { Controls } \\
(\mathbf{n = 3 2})\end{array}$ & $\begin{array}{c}\text { Cases } \\
(\mathbf{n = 3 2})\end{array}$ & $\boldsymbol{p}$-value \\
\hline Age (years), Mean $\pm \mathrm{SD}$ & $53.75 \pm 11.96$ & $55.71 \pm 10.27$ & 0.674 \\
Gender (male/female), n & $24 / 8$ & $25 / 7$ & - \\
Microalbumin (mg/L), Mean \pm SD & $7.84 \pm 5.07$ & $70.8 \pm 51.89$ & $<0.001$ \\
FBS (mg/dL), Mean \pm SD & $189.96 \pm 95.50$ & $186.07 \pm 50.37$ & 0.177 \\
HbA1c (g \%), Mean \pm SD & $8.89 \pm 3.01$ & $8.78 \pm 1.68$ & 0.39 \\
Creatinine (mg/dL), Mean \pm SD & $0.92 \pm 0.22$ & $0.91 \pm 0.36$ & 0.823 \\
\hline
\end{tabular}

equations were given in Table 3 and Table 4. LDL-C /LDL apoB showed a moderate negative association with apoB in cases $(\mathrm{r}=-0.666)$ and a weakly negative correlation in controls $(\mathrm{r}=-0.477)$. Likewise a weak negative correlation with $\mathrm{HbA1c}$ was seen in cases $(\mathrm{r}=-0.463)$. TG/HDL showed a weakly positive correlation with creatinine $(\mathrm{r}=0.391)$ and a moderately positive correlation with TG $(\mathrm{r}=0.796)$ and VLDL ( $\mathrm{r}=0.765)$ in cases. A moderate negative correlation with HDL-C was seen in cases as well as controls $(r=-0.705)$. In addition, the control group showed a moderately positive association with $\mathrm{FBS}(\mathrm{r}=0.599)$ and $\mathrm{HbAlc}$ $(\mathrm{r}=0.531)$. sdLDL-C showed a strong positive correlation with TC ( $\mathrm{r}=0.865)$, LDL-C $(\mathrm{r}=0.857)$ and non-HDL-C; $(\mathrm{r}=0.928)$. However a weak positive correlation was seen with TG $(r=0.490)$ and VLDL $(r=0.437)$. Similar finding was observed in the control group also (Table 3 and Table 4).

Figure 1 depicted the ROC curve for the evaluation of the above equations. Table 5 showed the values of the Area under the curve (AUC), sensitivity \& specificity of the equations. The AUC for LDL-C/LDL apoB was the highest with a sensitivity and specificity of $85 \%$ and $69 \%$ respectively.

\section{Discussion}

Epidemiological studies have reported increased sdLDL-C level that is closely associated with increased risk of CVD and cerebrovascular disease. $(5,21)$ Studies have demonstrated that many patients with CVD had LDL-C values in the same range as compared to the healthy subjects.(22) The present study did not show any significant difference in sdLDL-C levels in the diabetic subjects with and without nephropathy. This finding is in agreement with one of the earlier studies.(9) The high prevalence of sdLDL-C in T2DM with nephropathy is not directly associated with kidney damage.(23) A study on diabetic subjects with overt nephropathy have shown an increase in sdLDL-C in comparison with control subjects.(24) The

Table 2. Comparison of extended lipid profile among the subjects.

\begin{tabular}{lccc}
\hline \multicolumn{1}{c}{ Variable } & \multicolumn{2}{c}{ Mean \pm SD } & \\
\cline { 2 - 3 } & $\begin{array}{c}\text { Controls } \\
(\mathbf{n = 3 2})\end{array}$ & $\begin{array}{c}\text { Cases } \\
(\mathbf{n = 3 2})\end{array}$ & -value \\
\hline TC (mg/dL) & $180.27 \pm 39.82$ & $179.32 \pm 40.35$ & 0.993 \\
HDL-C (mg/dL) & $140.06 \pm 50.92$ & $146.78 \pm 51.33$ & 0.658 \\
LDL-C (mg/dL) & $43.62 \pm 12.55$ & $48.28 \pm 14.37$ & 0.478 \\
TC/HDL-C & $129.22 \pm 39.92$ & $120.61 \pm 37.54$ & 0.632 \\
TG (mg/dL) & $4.38 \pm 1.35$ & $3.90 \pm 1.10$ & 0.333 \\
VLDL (mg/dL) & $28.013 \pm 10.18$ & $29.82 \pm 10.18$ & 0.704 \\
Non HDL-C (mg/dL) & $134.95 \pm 37.57$ & $131.03 \pm 35.55$ & 0.753 \\
Apo B (mg/dL) & $115.90 \pm 45.00$ & $101.38 \pm 37.76$ & 0.122 \\
LDL-C/LDL apo B & $1.76 \pm 1.54$ & $1.84 \pm 0.92$ & 0.787 \\
sdLDL-C (mg/dL) & $41.92 \pm 12.91$ & $39.93 \pm 12.12$ & 0.48 \\
TG/HDL-C & $3.59 \pm 1.96$ & $3.39 \pm 1.75$ & 0.62 \\
\hline
\end{tabular}


Table 3. Correlations of clinical parameters with the equations in the case group.

\begin{tabular}{|c|c|c|c|c|}
\hline \multicolumn{2}{|c|}{ Variable } & TG/HDL & LDL-C/LDL apo B & sdLDL-C \\
\hline \multirow{2}{*}{ Age } & $\mathrm{r}$ & 0.282 & 0.066 & 0.092 \\
\hline & $\mathrm{p}$ & 0.146 & 0.738 & 0.643 \\
\hline \multirow{2}{*}{ FBS } & $\mathrm{r}$ & -0.078 & -0.321 & 0.026 \\
\hline & $\mathrm{p}$ & 0.693 & 0.096 & 0.897 \\
\hline \multirow{2}{*}{ HbAlc } & $\mathrm{r}$ & -0.16 & -0.463 & -0.009 \\
\hline & $\mathrm{p}$ & 0.415 & $0.013^{*}$ & 0.963 \\
\hline \multirow{2}{*}{ Microalbumin } & $\mathrm{r}$ & -0.154 & -0.045 & -0.148 \\
\hline & $\mathrm{p}$ & 0.435 & 0.819 & 0.452 \\
\hline \multirow{2}{*}{ Creatinine } & $\mathrm{r}$ & 0.391 & -0.089 & -0.301 \\
\hline & $\mathrm{p}$ & $0.040^{*}$ & 0.651 & 0.12 \\
\hline \multirow{2}{*}{$\mathrm{TC}$} & $\mathrm{r}$ & -0.195 & 0.114 & 0.865 \\
\hline & $\mathrm{p}$ & 0.321 & 0.564 & $<0.001^{*}$ \\
\hline \multirow{2}{*}{ TG } & $\mathrm{r}$ & 0.796 & 0.006 & 0.49 \\
\hline & $\mathrm{p}$ & $<0.001^{*}$ & 0.977 & $<0.01^{*}$ \\
\hline \multirow{2}{*}{ HDL } & $\mathrm{r}$ & -0.705 & 0.225 & 0.135 \\
\hline & $\mathrm{p}$ & $.000 *$ & 0.25 & 0.494 \\
\hline \multirow{2}{*}{ LDL-C } & $\mathrm{r}$ & -0.192 & 0.04 & 0.857 \\
\hline & $\mathrm{p}$ & 0.329 & 0.84 & $<0.001 *$ \\
\hline \multirow{2}{*}{ VLDL } & $\mathrm{r}$ & 0.765 & -0.017 & 0.437 \\
\hline & $\mathrm{p}$ & $<0.001^{*}$ & 0.932 & $0.02 *$ \\
\hline \multirow{2}{*}{ Non HDL } & $\mathrm{r}$ & 0.064 & 0.038 & 0.928 \\
\hline & $\mathrm{p}$ & 0.746 & 0.847 & $<0.001 *$ \\
\hline \multirow{2}{*}{ Apo B } & $\mathrm{r}$ & -0.102 & -0.666 & 0.509 \\
\hline & $\mathrm{p}$ & 0.607 & $.000 *$ & $<0.01 *$ \\
\hline
\end{tabular}

Weak correlation: $\mathrm{r}= \pm 0.3$ to 0.5 ; moderate correlation: $\mathrm{r}= \pm 0.5$ to 0.8 ; strong correlation: $\mathrm{r}= \pm 0.8$ to 1 . Correlation is significant when $p \leq 0.05$.

values of sdLDL-C reported is in agreement with the values obtained in the present study. The sdLDL-C values obtained in the present study are in agreement with the previous study which found that the link of sdLDL-C to diabetes is considered to be through insulin resistance. $(25,26)$ The present study did not show any association of sdLDL-C with FBS and HbA1c. Average FBS was significantly higher in patients with microalbuminuria in comparison with the normal subjects.(27) Cholesterol carried in sdLDL-C was significantly higher in severe CHD and its concentration was associated with severity of coronary atherosclerosis independent of the levels of LDL-C, apoB, non-HDL and HDL-C in patients with CVD.(28) Even though sdLDL-C has been shown to be a good biomarker for assessing the response to therapeutic interventions in T2DM patients with dyslipidemia sdLDL-C seems to be an independent risk factor for CVD because patients with CVD may have LDL-C within the reference intervals but increased levels of sdLDL-C.(22, 29-30)

Subjects with metabolic syndrome had a TG/HDL ratio that was 2 times higher compared to the controls (5.10 vs. 2.03) and was associated to the first coronary event.(31) No significant change in the TG/HDL ratio was observed in the two groups of the present study (3.59vs. 3.39). A study reported that $75 \%$ of the subjects with small LDL size $(<25.5$ $\mathrm{nm}$ ) had TG/HDL ratios higher than 0.9 while only $25 \%$ of the subjects with normal LDL size $(>25.5 \mathrm{~nm})$ had TG/HDL ratios above 0.9.(32) TG/HDL-C ratio is anticipated to be beneficial for assessing the effects of various therapies aimed at preventing small LDL formation. 
Table 4. Correlations of clinical parameters with the equations in the control group.

\begin{tabular}{|c|c|c|c|c|}
\hline \multicolumn{2}{|c|}{ Variable } & \multirow{2}{*}{$\begin{array}{c}\text { TG/HDL } \\
-0.379\end{array}$} & \multirow{2}{*}{$\begin{array}{c}\text { LDL-C/LDL apo B } \\
-0.06\end{array}$} & \multirow{2}{*}{$\begin{array}{c}\text { sdLDL-C } \\
-0.274\end{array}$} \\
\hline$\Delta \alpha$ & $\mathrm{r}$ & & & \\
\hline Igo & $\mathrm{p}$ & $0.043^{*}$ & 0.758 & 0.15 \\
\hline \multirow{2}{*}{ FBS } & $\mathrm{r}$ & 0.599 & -0.076 & 0.186 \\
\hline & $\mathrm{p}$ & $0.001^{*}$ & 0.694 & 0.334 \\
\hline \multirow{2}{*}{$\mathrm{HbAlc}$} & $\mathrm{r}$ & 0.531 & -0.14 & 0.217 \\
\hline & $\mathrm{p}$ & $0.003^{*}$ & 0.468 & 0.258 \\
\hline \multirow{2}{*}{ Microalbumin } & $\mathrm{r}$ & -0.108 & 0.243 & -0.315 \\
\hline & $\mathrm{p}$ & 0.576 & 0.204 & 0.096 \\
\hline \multirow{2}{*}{ Creatinine } & $\mathrm{r}$ & -0.307 & 0.082 & -0.305 \\
\hline & $\mathrm{p}$ & 0.106 & 0.672 & 0.108 \\
\hline \multirow{2}{*}{$\mathrm{TC}$} & $\mathrm{r}$ & -0.094 & 0.214 & 0.869 \\
\hline & $\mathrm{p}$ & 0.629 & 0.265 & $<0.001 *$ \\
\hline \multirow{2}{*}{ TG } & $\mathrm{r}$ & 0.834 & 0.095 & 0.461 \\
\hline & $\mathrm{p}$ & $<0.001 *$ & 0.623 & $0.012 *$ \\
\hline \multirow{2}{*}{ HDL-C } & $\mathrm{r}$ & -0.709 & -0.012 & -0.122 \\
\hline & $\mathrm{p}$ & $<0.001 *$ & 0.95 & 0.527 \\
\hline \multirow{2}{*}{ LDL-C } & $\mathrm{r}$ & -0.044 & 0.193 & 0.875 \\
\hline & $\mathrm{p}$ & 0.82 & 0.317 & $<0.001 *$ \\
\hline \multirow{2}{*}{ VLDL } & $\mathrm{r}$ & 0.834 & 0.095 & 0.461 \\
\hline & $\mathrm{p}$ & $0.001^{*}$ & 0.623 & $0.01 *$ \\
\hline \multirow{2}{*}{ Non HDL-C } & $\mathrm{r}$ & 0.184 & 0.25 & 0.896 \\
\hline & $\mathrm{p}$ & 0.34 & 0.191 & $<0.001 *$ \\
\hline \multirow{2}{*}{ Apo B } & $\mathrm{r}$ & 0.102 & -0.477 & 0.568 \\
\hline & $\mathrm{p}$ & 0.597 & $<0.01 *$ & $0.001 *$ \\
\hline
\end{tabular}

Weak correlation: $\mathrm{r}= \pm 0.3$ to 0.5 ; moderate correlation: $\mathrm{r}= \pm 0.5$ to 0.8 ; strong correlation: $\mathrm{r}= \pm 0.8$ to 1 . Correlation is significant when $p \leq 0.05$.

The sdLDL-C showed strong correlation with TC, LDL-C and non HDL-C and a moderate positive correlation with TG and VLDL in both the groups of the present study. On the contrary, a positive correlation of sdLDL-C with the TG and LDL-C and a negative correlation with HDL-C values were observed in diabetic subjects with and without nephropathy.(33) However, negative association with HDL was not seen in the present study. LDL-C/LDL apoB showed negative association with apo $\mathrm{B}$ in both cases and controls whereas a negative association with FBS and $\mathrm{HbA} 1 \mathrm{c}$ is seen only in cases. However no correlation was found with any of the traditional risk factors. It has been shown that the LDL particle size measured by gradient gel electrophoresis did not match with that of LDL-C/LDL apoB ratio.(34)
The equation for calculation of sdLDL-C was not useful in specific patient group such as kidney disease, diabetes mellitus, metabolic syndrome, etc.(21) There was no significant difference in the calculated sdLDL-C levels in the groups with and without nephropathy. The present study was not able to differentiate diabetic patients with and without nephropathy from the calculated sdLDL-C levels. However, it can have an important role in helping the clinicians to embrace the calculated sdLDL-C cholesterol as an additional approach for the assessment of CVD risk.

The cut off value obtained for TG/HDL (3.0) and LDL-C/ LDL apoB (1.2) ARE in agreement with the values reported in the earlier studies.(20) The cut off value for calculated sdLDL-C was found to be 30.5 from our study. These 


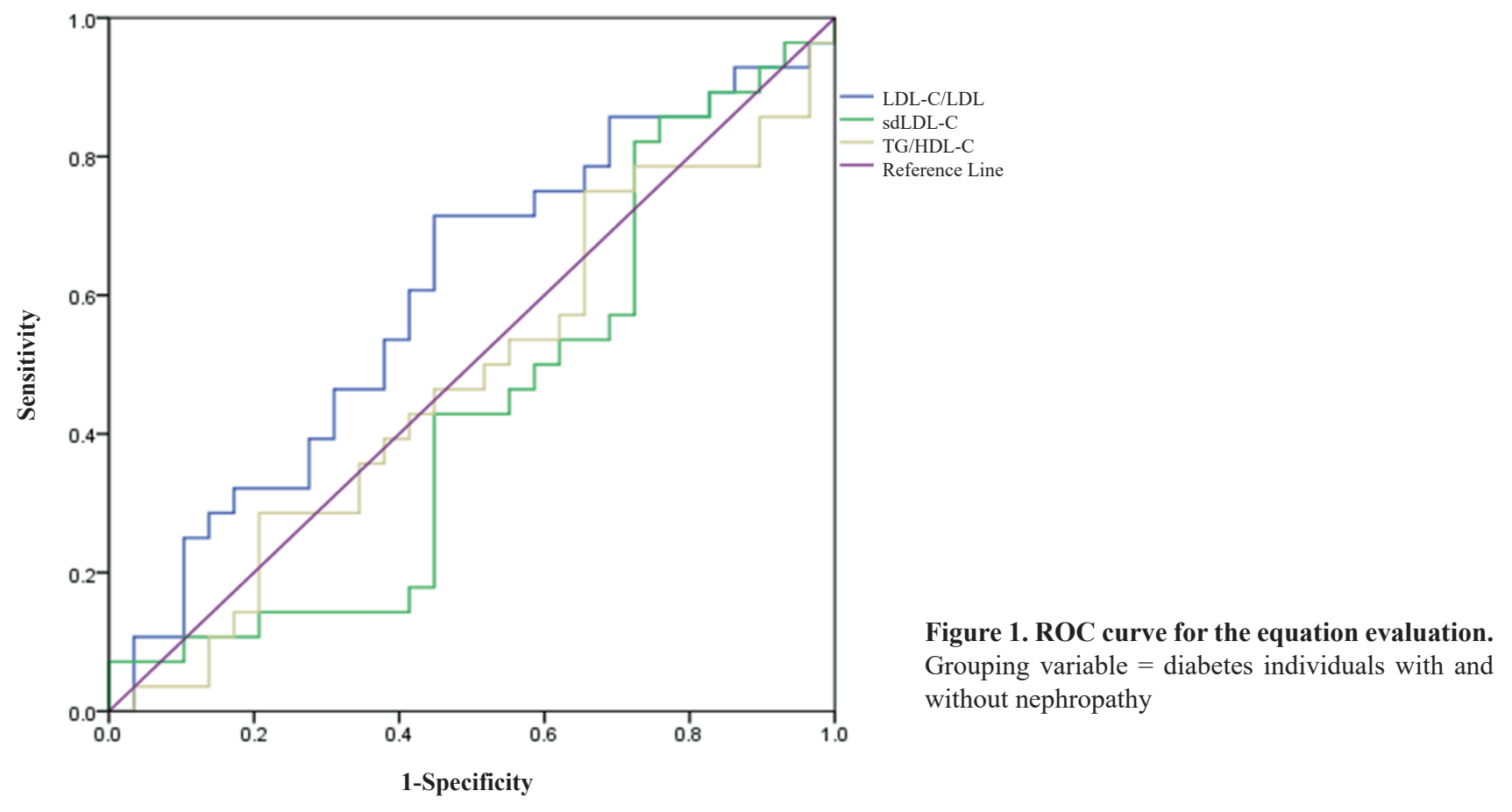

Table 5. Values of AUC based on the ROC curve.

\begin{tabular}{lccc}
\hline \multicolumn{1}{c}{ Variable } & Area & Sensitivity & Specificity \\
\hline LDL-C/LDLapo B & 0.6 & $85 \%$ & $69 \%$ \\
sdLDL-C & 0.436 & $82 \%$ & $75 \%$ \\
TG/HDL & 0.478 & $46 \%$ & $44 \%$ \\
\hline
\end{tabular}

findings suggest the usefulness of these equations in predicting sd LDL-C levels in diabetes, CAD and kidney diseases.

Of the three equations, LDL-C/LDL apo B appears to be a better predictor of sdLDL-C measurement because of its higher sensitivity. Moreover, sdLDL-C measured by calculation is an inexpensive cost effective method which can be used as a surrogate marker for the assessment of CVD risk. Due to the short duration of the study period, the number of samples studied were less. Future studies with a larger sample size and extended study period may show a better outcome.

\section{Conclusion}

The study showed that calculated sdLDL-C does not predict the evidence of nephropathy in diabetics with and without microalbuminuria. However, measurement of sdLDL-C by calculation may still be considered as a potential risk factor to be used in conjunction with the traditional markers since it is cost effective and quantifiable unlike TG/HDL-C and LDL-C/LDL apoB which are categorical. The formula LDL-C/LDL apo $\mathrm{B}$ is a best predictor of sdLDL among the three equations studied.

\section{Acknowledgements}

The authors are grateful to the Department of Microbiology KMCHAC, Mangalore for the assistance, given in the estimation of apo B.

\section{References}

1. Martín-Timón I, Sevillano-Collantes C, Segura-Galindo A, Del Cañizo-Gómez FJ. Type 2 diabetes and cardiovascular disease: Have all risk factors the same strength?. World J Diabetes. 2014; 5: 444-70.

2. Viazzi F, Leoncini G, Conti N, Tomolillo C, Giachero G, Vercelli M, 
et al. Microalbuminuria is a predictor of chronic renal insufficiency in patients without diabetes and with hypertension: the MAGIC study. Clin J Am SocNephrol. 2010; 5: 1099-106.

3. Gerber PA, Thalhammer C,Schmied C, Spring S, Amann-Vesti B, Spinas GA, et al. Small, dense LDL particles predict changes in intima media thickness and insulin resistance in men with type 2 diabetes and prediabetes - A prospective cohort study. PloS ONE. 2013; 8: e72763. doi: 10.1371/journal.pone.0072763.

4. Fan J, Liu Y, Yin S,hen N, Bai X, Ke Q, Shen J, et al. Small dense LDL cholesterol is associated with metabolic syndrome traits independently of obesity and inflammation. NutrMetab (Lond). 2019; 16: 7. doi: 10.1186/s12986-019-0334-y.

5. Goel PK, Ashfaq F, Khanna R, Ramesh V, Pandey CM. The association between small dense low density lipoprotein and coronary artery disease in North Indian patients. Indian J Clin Biochem. 2017; 32: 186-92.

6. Xia F, Liu G, Shi Y, Zhang Y. Impact of microalbuminuria on incident coronary heart disease, cardiovascular and all-cause mortality: a meta-analysis of prospective studies. Int J Clin Exp Med. 2015; 8: $1-9$.

7. Ivanova EA, Myasoedova VA, Melnichenko AA, Grechko AV, Orekhov AN. Small dense low-density lipoprotein as biomarker for atherosclerotic diseases. Oxid Med Cell Longev. 2017; 2017: 1273042. doi: $10.1155 / 2017 / 1273042$.

8. Srisawasdi P, Chaloeysup S, Teerajetgul Y, Pocathikorn A, Sukasem $\mathrm{C}$, Vanavanan $\mathrm{S}$, et al. Estimation of plasma small dense LDL cholesterol from classic lipid measures. Am J Clin Pathol. 2011; 136: 20-9.

9. Ito Y, Fujimura M, Ohta M, Hirano T. Development of a homogeneous assay for measurement of small dense LDL cholesterol.Clin Chem. 2011; 57: 57-65.

10. Koga M, Okuda M, Inada S, Shin-Ichiro U, Yuko N, Toshika O, et al. $\mathrm{HbA1c}$ levels measured by enzymatic assay during off-site health checkups are lower than those measured by on-site HPLC assay. Diabetol Int. 2019; 11: 67-71.

11. Stefani S, Ngatidjan S, Paotiana M, Sitompul KA, Abdullah M, Sulistianingsih DP, et al. Dietary quality of predominantly traditional diets is associated with blood glucose profiles, but not with total fecal Bifidobacterium in Indonesian women. PLoS One. 2018; 13: e0208815. doi: 10.1371/journal.pone.0208815.

12. Corso G, Papagni F, Gelzo M, Gallo M, Barone R, Graf M, et al. Development and validation of an enzymatic method for total cholesterol analysis using whole blood spot. J Clin Lab Anal. 2016; 30: $517-23$.

13. Rohatgi R, Gupta R, Ray R, Kalra V. Is obstructive sleep apnea the missing link between metabolic syndrome and second-generation antipsychotics: Preliminary study. Indian J Psychiatry. 2018; 60: 478-84.

14. Fawwad A, Sabir R, Riaz M, Moin H, Basit A. Measured versus calculated LDL-cholesterol in subjects with type 2 diabetes. Pak J Med Sci. 2016; 32: 955-60.

15. Penumarthy S, Penmetsa GS, Mannem S. Assessment of serum levels of triglycerides, total cholesterol, high-density lipoprotein cholesterol, and low-density lipoprotein cholesterol in periodontitis patients. J Indian Soc Periodontol. 2013; 17: 30-5.

16. Hoste L, Deiteren K, Pottel H, Callewaert N, Martens F. Routine serum creatinine measurements: how well do we perform? BMC Nephrol. 2015; 16: 21. doi: 10.1186/s12882-015-0012-x.

17. Marcovina SM, Albers JJ. Lipoprotein (a) measurements for clinical application. J Lipid Res. 2016; 57: 526-37.
18. Swetha NK, Virupaksha HS, Vishwanath P, Suma MN, Bhimappa M. Hypomagnesemia, poor glycemic control and microalbuminuria as risk factors of diabetic retinopathy. Natl J Lab Med. 2014; 3: 10-4.

19. Palazhy S, Kamath P, Vasudevan DM. Estimation of small, dense LDL particles using equations derived from routine lipid parameters as surrogate markers. Biochem Anal Biochem. 2014; 3: 1000146. doi: 10.4172/2161-1009.1000146.

20. Maruyama C, Imamura K, Teramoto T. Assessment of LDL particle size by triglyceride/HDL-cholesterol ratio in non-diabetic, healthy subjects without prominent hyperlipidemia. J Atheroscler Thromb. 2003; 10: 186-91.

21. Hirayama S, Miida T. Small dense LDL: An emerging risk factor for cardiovascular disease. Clin Chim Acta. 2012; 414: 215-24.

22. Krittayaphong R, Phrommintikul A, Boonyaratvej S, Na Ayudhya RK, Tatsanavivat $\mathrm{P}$, Komoltri $\mathrm{C}$, et al. The rate of patients at high risk for cardiovascular disease with an optimal low-density cholesterol level: a multicenter study from Thailand. J Geriatr Cardiol. 2019; 16: 344-53.

23. Essam A, Badr HB, Mohamad A, Ahmad OM, Hamdy S. Small dense low-density lipoprotein as a potential risk factor of nephropathy in type 2 diabetes mellitus. Indian J Endocrinol Metab. 2014; 18: 94-8.

24. Palazhy S, Viswanathan V. Lipid abnormalities in type 2 diabetes mellitus patients with overt nephropathy. Diabetes Metab J. 2017; 41: $128-34$

25. Hirano T. Pathophysiology of diabetic dyslipidemia. J Atheroscler Thromb. 2018; 25: 771-82.

26. Klop B, Elte JWF, Cabezas MC. Dyslipidemia in obesity: Mechanisms and potential targets. Nutrients. 2013; 5: 1218-40.

27. Pasko N, Toti F, Strakosha A, Thengjilli E, Shehu A, Dedej T, et al. Prevalence of microalbuminuria and risk factor analysis in type 2 diabetes patients in Albania: the need for accurate and early diagnosis of diabetic nephropathy. Hippokratia. 2013; 17: 337-41.

28. Manjunath $\mathrm{CN}$, Rawal JR, Irani PM, Madhu K. Atherogenic dyslipidemia. Indian J Endocrinol Metab. 2013; 17: 969-76.

29. Karupaiah T, Chuah KA, Chinna K, Pressman P, Clemens RA, Hayes AW, et al. A cross-sectional study on the dietary pattern impact on cardiovascular disease biomarkers in Malaysia. Sci Rep. 2019; 9: 13666. doi: 10.1038/s41598-019-49911-6.

30. He J, He S, Liu K, Wang Y, Shi D, Chen X. The TG/HDL-C ratio might be a surrogate for insulin resistance in Chinese nonobese women. Int J Endocrinol. 2014; 2014: 105168. doi: $10.1155 / 2014 / 105168$.

31. Ren X, Chen Z, Zheng S, Han T, Li Y, Liu W, et al. Association between triglyceride to HDL-C Ratio (TG/HDL-C) and insulin resistance in Chinese patients with newly diagnosed type 2 diabetes mellitus. PLoS ONE. 2016; 11: e0154345. doi: 10.1371/journal. pone. 0154345 .

32. Abd-Allha E, Hassan BB, Abduo M, Omar SA, Sliem H. Small dense low-density lipoprotein as a potential risk factor of nephropathy in type 2 diabetes mellitus. Indian J Endocrinol Metab. 2014; 18: 94-8.

33. Tani S, Yagi T, Atsumi W, Kawauchi K, Matsuo R, Hirayama A. Relation between low-density lipoprotein cholesterol/ apolipoprotein B ratio and triglyceride-rich lipoproteins in patients with coronary artery disease and type 2 diabetes mellitus: a crosssectional study. CardiovascDiabetol. 2017; 16: 123. doi: 10.1186/ s12933-017-0606-7.

34. Wakabayashi I, Daimon T. Comparison of discrimination for cardiometabolic risk by different cut-off values of the ratio of triglycerides to HDL cholesterol. Lipids Health Dis. 2019; 18: 156. doi: 10.1186/ s12944-019-1098-0 